\title{
Efeitos da deficiência de vitamina A em fetos de gestantes após derivação gástrica em $Y$ de Roux: revisão integrativa
}

\author{
Effects of vitamin A deficiency in fetuses post Roux-en-Y gastric bypass pregnancy: \\ integrative review
}

\section{Efectos de la deficiencia de vitamina $A$ en fetos de mujeres embarazadas después de la derivación gástrica en $Y$ de Roux: revisión integradora}

Thais Helena Carreira Cotillo ${ }^{1}$, Priscilla Sampaio ${ }^{1}$, Priscila Sala Kobal ${ }^{1 *}$.

\begin{abstract}
RESUMO
Objetivo: Identificar a frequência de deficiência da vitamina A em gestantes após a Derivação Gástrica em $Y$ de $R o u x$ (DGYR) e avaliar os efeitos nos fetos. Métodos: Revisão integrativa de literatura baseada em artigos publicados nas bases de dados eletrônicas PubMed/MEDLINE, Scientific Eletronic Library Online (Scielo), Web of Science e SCOPUS (Elsevier). Os descritores usados foram "vitamina A", "deficiência de vitamina A", "gestante" e "Derivação Gástrica em $Y$ de Roux". Apenas artigos escritos no idioma português e inglês, na íntegra, indexados nas referidas bases de dados, e publicados dentre os anos de 2016 e 2021, foram incluídos no estudo. Resultados: Foram selecionados doze artigos. Os resultados obtidos relataram que há um aumento na deficiência de vitamina A após a DGYR, e quando associado a gestação após a cirurgia, pode ocorrer o agravamento dessa deficiência. Em fetos e recém-nascidos, de mães que passaram pela cirurgia, foram identificados deficiência de vitamina A no cordão umbilical, malformações congênitas, alteração na microbiota intestinal e consequências na imunidade. Considerações Finais: Gestação após a DGYR aumenta a ocorrência de deficiência de vitamina A. O monitoramento nutricional é recomendado em prol da saúde materna e fetal, antes e após a cirurgia, e durante a gravidez.
\end{abstract}

Palavras-chave: Cirurgia bariátrica, Deficiência de vitamina A, Gestantes, Feto.

\begin{abstract}
Objective: Identify the frequency of vitamin A deficiency in pregnant women post Roux-en-Y gastric bypass (RYGP) and to assess the effects on fetuses. Methods: Integrative literature review based on articles publishes in PubMed/MEDLINE, Scientific Eletronic Library Online (Scielo), Web of Science e SCOPUS (Elsevier), as electronic data bases. The descriptors used were 'vitamin A', 'vitamin A deficiency', 'pregnancy', and 'Roux-en- $Y$ gastric bypass'. Only Portuguese and English language full-text article, indexed in the aforementioned data bases, published between 2016 and 2021 were included within this study. Results: Twelve articles were selected. As results, it was reported an increase in vitamin A deficiency after RYGP, and when associated with pregnancy post-surgery, may aggravate the deficiency. In fetuses and newborn, of post-surgery mothers, identified vitamin A deficiency in the umbilical cord, congenital malformation, alteration in the intestinal microbiota and consequences on immune system. Final Considerations: Pregnancy post RYGB increases the occurrence of vitamin A deficiency. Nutritional follow-up is necessary for maternal and fetal health previous and post-surgery, and during pregnancy.
\end{abstract}

Keywords: Bariatric surgery, Vitamin A deficiency, Pregnant women, Fetus.

\section{RESUMEN}

Objetivo: Identificar la frecuencia de deficiencia de vitamina A en mujeres embarazadas después del Derivación Gástrica en $Y$ de Roux (DGYR) y evaluar los efectos en los fetos. Métodos: Revisión integrativa de la literatura basada en artículos publicados en las bases de datos electrónicas PubMed/MEDLINE, Scientific Electronic Library Online (Scielo), Web of Science y SCOPUS (Elsevier). Los descriptores utilizados fueron "vitamina A", "deficiencia de vitamina A", "mujer embarazada" y "derivación gástrica en Y de Roux". Solo se incluyeron en el estudio artículos escritos en portugués e inglés, en su totalidad, indexados en estas bases de datos y publicados entre 2016 y 2021. Resultados: Se seleccionaron doce artículos. Los resultados obtenidos informaron que hay un aumento de la deficiencia de vitamina A después de la DGYR, y cuando se asocia con el embarazo después de la cirugía, esta deficiencia puede empeorar. En fetos y recién nacidos de madres operadas, se identificaron deficiencia de vitamina A en el cordón umbilical, malformaciones congénitas, cambios en la microbiota intestinal y consecuencias sobre la inmunidad. Consideraciones finales: El embarazo después de la DGYR aumenta la aparición de deficiencia de vitamina A. Se recomienda el control nutricional para el beneficio de la salud materna y fetal, antes y después de la cirugía y durante el embarazo.

Palabras clave: Cirugía bariátrica, Deficiencia de vitamina A, Mujeres embarazadas, Feto.

${ }^{1}$ Centro Universitário São Camilo, São Paulo - SP. *E-mail: priscila.kobal@saocamilo-sp.br 


\section{INTRODUÇÃO}

Obesidade é definida como acúmulo excessivo de gordura corporal sendo classificada pelo Índice de Massa Corporal (IMC) $\geq 30 \mathrm{Kg} / \mathrm{m}^{2}$ (WORLD HEALTH ORGANIZATION (WHO), 2020). A prevalência global de obesidade em 2025 chegará a $21 \%$ nas mulheres e 18\% nos homens e a obesidade severa ultrapassará $9 \%$ nas mulheres e $6 \%$ nos homens (NCD-RISC, 2016). Um dos impactos da obesidade é o risco de subfertilidade (BROUGHTON DE e MOLEY KH, 2017).

Mulheres obesas normalmente apresentam irregularidade menstrual como distúrbios ovulatórios, patologia endometrial e infertilidade, evidenciando que a obesidade ocasiona efeitos negativos na fisiologia reprodutiva (SILVESTRIS E, et al., 2018). A obesidade também está associada a resultados negativos para pacientes que realizam fertilização in vitro devido à baixa qualidade do oócito, menor taxa de pré-implantação e receptividade uterina (BELLVER J, et al., 2010).

Foi demonstrado que a perda de peso pode potencializar a melhora na reprodução em mulheres (WISE LA, et al., 2013). A cirurgia bariátrica antes da gestação é promissora para reduzir as comorbidades maternas relacionadas à obesidade, incluindo diabetes gestacional e pré-eclâmpsia (AKHTER Z, et al., 2019). Além de resultar na perda de peso, reduz a prevalência da síndrome metabólica e melhora a resistência à insulina e o hiperandrogenismo. Restaurando os ciclos menstruais regulares e auxiliando no sucesso da gestação (LEE $R$, et al., 2020).

Dentre os procedimentos mais realizados no mundo, a derivação gástrica se destaca contabilizando nos Estados Unidos mais de 60 a 70\% de todas as cirurgias bariátricas desde 2003 (MITCHELL BG e GUPTA N, 2020). Segundo dados da International Federation for the Surgery of Obesity and Metabolic Disorders (IFSO), entre os anos 2014 a 2018, em 51 países dos 5 continentes, foi registrado que a Derivação Gástrica em Y de Roux (DGYR) é o segundo procedimento cirúrgico mais executado no mundo correspondendo a $38,2 \%$ de todas as técnicas realizadas, logo após a gastrectomia vertical (sleeve) que atualmente é a técnica mais executada, apresentando um percentual de 46\% (WELBOURN R, et al., 2019).

A DGYR trata-se de uma reconstrução do trânsito Gastrointestinal (GI) em que se cria uma pequena bolsa gástrica com um volume de $10-50 \mathrm{~mL}$, promovendo o desvio do alimento da maior parte do estômago e parte proximal do intestino delgado por meio de anastomose. Após cerca de $150 \mathrm{~cm}$, outra anastomose é feita da alça biliopancreática no jejuno. Como resultado, a maior parte do estômago, o duodeno e o jejuno proximal são excluídos do trânsito alimentar. Sendo assim, a DGYR é um procedimento misto em que se reduz o espaço disponível para o alimento e ocasionando uma menor ingestão. Com o desvio do intestino proximal, há a promoção do aumento de hormônios, que proporcionam à saciedade e redução da fome, além de uma absorção alimentar prejudicada. Esses mecanismos colaboram com o emagrecimento (SBCBM, 2017).

Apesar dos benefícios, é comum ocorrer deficiências nutricionais no pós-operatório, devido ao prejuízo na absorção de vitaminas e nutrientes essenciais (HARRISON RK, et al., 2018, CUESTA M, et al., 2014). Quando se associa a DGYR à gestação, observa-se mais de $75 \%$ de inadequação de vitamina $A$, concluindo que a gestação após a cirurgia pode representar uma situação de alto risco para deficiências (CRUZ S, et al., 2018; MACHADO SN, et al., 2016).

A vitamina A é micronutriente essencial, responsável por diversos funcionamentos do organismo, como visão, expressão gênica, manutenção da integridade celular epitelial, fortalecimento do sistema imune e crescimento e diferenciação celular (YONEKURA L, et al., 2020). Tal hipovitaminose, durante a gestação, pode ocasionar desfechos negativos para a mãe e para o feto, como cegueira noturna, doenças infecciosas, baixo peso ao nascer e problemas na maturação pulmonar (MARTIN APR e MASQUIO DCL, 2019; AGARWAL R, et al., 2013; CHECKLEY WJ, et al., 2010, TIMONEDA J, et al., 2018).

O objetivo desse trabalho foi identificar a frequência de deficiência de vitamina A em gestantes após DGYR e avaliar os efeitos nos fetos.

\section{MÉTODOS}

O presente trabalho trata-se de uma revisão integrativa da literatura baseada na análise de artigos referentes a deficiência de vitamina A em gestantes após DGYR e os efeitos no feto. Para o desenvolvimento dessa revisão, foram utilizadas as seguintes etapas: escolha do tema de estudo, escolha das bases de dados, elaboração dos critérios de inclusão e exclusão e análise e interpretação dos resultados. 
A pesquisa foi realizada entre outubro de 2020 e dezembro de 2021, simultaneamente por dois pesquisadores. Para busca e seleção de artigos, foram consultadas as bases de dados eletronicas PubMed/MEDLINE, Scientific Eletronic Library Online (Scielo), Web of Science e SCOPUS (Elsevier) e foram utilizados os descritores controlados da Biblioteca Virtual em Saúde por meio dos Descritores em Ciências da Saúde (Decs) constando de "vitamina A", "deficiência de vitamina A", "gestante" e "Derivação Gástrica em Y de Roux", e dos Medical Subject Headings (Mesh) constando "vitamin A", "deficiency of vitamin A", "pregnancy" e "Roux en Y Gastric Bypass". Durante a busca foi utilizado o operador booleano "AND", uma vez que o mesmo favorece a intersecção no decorrer da procura.

Como critério de inclusão para o levantamento bibliográfico consideraram-se artigos disponíveis nas bases de dados supracitadas, na íntegra, no idioma inglês ou português, publicados entre os anos de 2016 e 2021, que retratassem temáticas como deficiência de vitamina A pós DGYR, gestação após DGYR e os efeitos da hipovitaminose A no desenvolvimento do feto. Artigos referentes a deficiência de outras vitaminas, pesquisas sobre outras condições fisiológicas que não abranja gestação, deficiência nutricional frente a outro procedimento de redução de peso que não seja após DGYR e nutrição pediátrica foram excluídos desse estudo.

\section{RESULTADOS}

Foram eleitos 12 artigos científicos sobre deficiência de vitamina A em gestantes após DGYR e os efeitos no feto. A princípio, forma encontrados 345 estudos, porém 145 foram excluídos por título inadequado para a referida pesquisa, 22 por duplicação. Em seguida, outros 166 artigos foram excluídos por não atenderem aos requerimentos de seleção previamente estabelecidos. Dos 12 trabalhos incluídos, 4 (a maioria) foram publicações do ano de 2018 (33,2\%), seguidos por $3(25 \%)$ publicados em 2017, por $2(16,7 \%)$ publicados em 2021 e por 1 estudo nos anos de 2016, 2019 e 2020 (8,3\%). A Figura 1 ilustra os procedimentos para seleção dos artigos eleitos.

Figura 1 - Fluxograma do processo de seleção dos artigos.

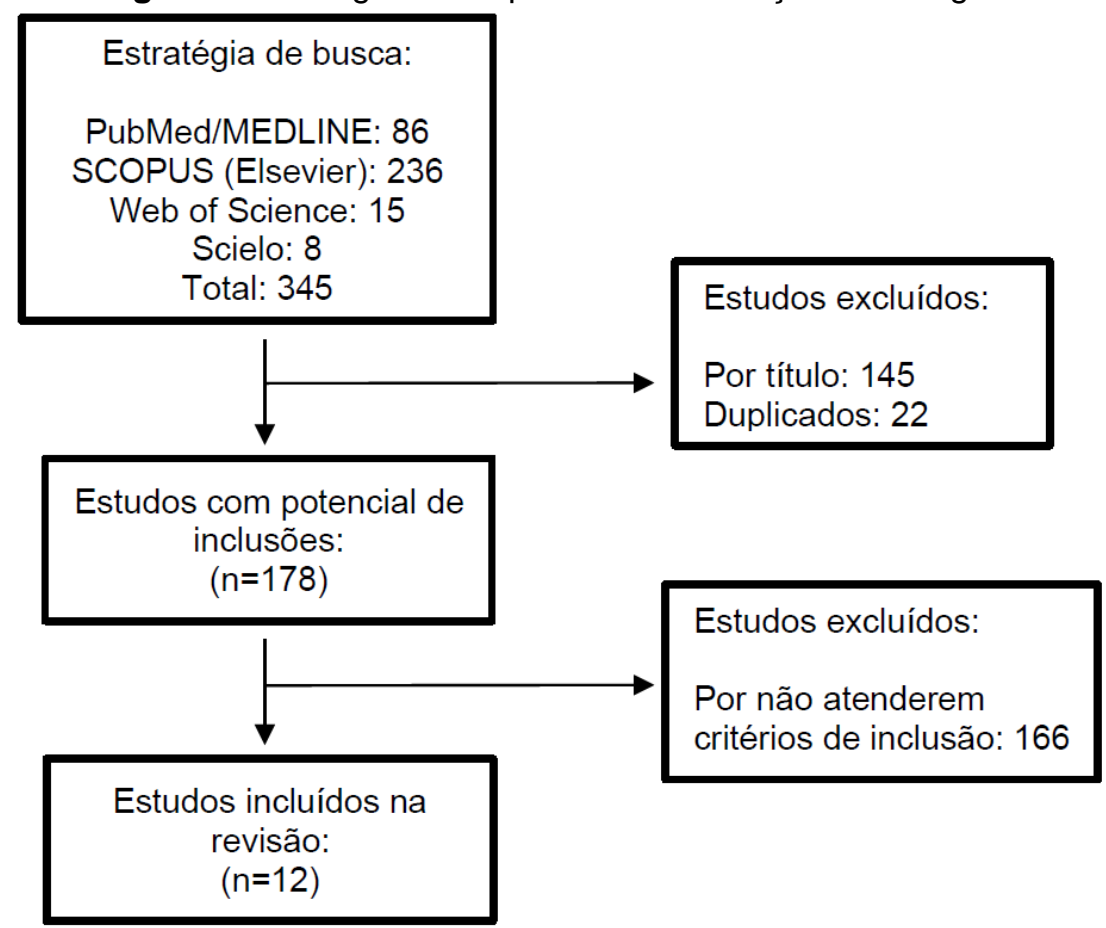

Fonte: Cotillo THC, et al., 2021.

No Quadro 1 são apresentados dados extraídos dos 12 artigos selecionados organizados segundo autoria, objetivos, principais resultados e conclusão. Além dos trabalhos suscitados no quadro abaixo, outros documentos contendo informações adicionais, que não faziam parte dos critérios de inclusão, foram utilizados na discussão afim de complementar a discussão e agregar informações a temática. 
Quadro 1 - Síntese dos artigos incluídos na revisão.

\begin{tabular}{|c|c|c|}
\hline AUTORIA & OBJETIVO & PRINCIPAIS RESULTADOS \\
\hline $\begin{array}{l}\text { MACHADO SN, } \\
\text { et al. (2016). }\end{array}$ & $\begin{array}{l}\text { Avaliar o estado nutricional da vitamina } A \\
\text { por meio de indicadores bioquímicos e } \\
\text { funcionais de gestantes submetidas à } \\
\text { Derivação Gástrica em Y de Roux (DGYR) } \\
\text { em comparação com gestantes não } \\
\text { submetidas a esta cirurgia. }\end{array}$ & $\begin{array}{l}\text { As médias do retinol sérico e do } \beta \text {-caroteno foram significativamente } \\
\text { maiores no grupo de gestantes não cirúrgicas em relação ao grupo de } \\
\text { gestantes que realizaram a DGYR. Em relação ao indicador funcional } \\
\text { para avaliação da deficiência de vitamina A, aproximadamente } 75 \% \\
\text { das gestantes pós DGYR apresentaram cegueira noturna e } 20 \% \text { das } \\
\text { gestantes, sendo que o percentual de gestantes com esse } \\
\text { comprometimento funcional foi significativamente maior no nas } \\
\text { gestantes pós DGYR em relação a gestantes. }\end{array}$ \\
\hline $\begin{array}{l}\text { GASCOIN G, et } \\
\text { al. (2017). }\end{array}$ & $\begin{array}{l}\text { Rastrear deficiências de micronutrientes } \\
\text { em recém-nascidos de mães com bypass } \\
\text { gástrico. }\end{array}$ & $\begin{array}{l}\text { Os recém-nascidos de mães que realizaram DGYR eram pequenos } \\
\text { para a idade gestacional em } 23 \% \text { dos casos contra } 3,6 \% \text { no grupo de } \\
\text { controle. Uma porcentagem maior de neonatos DGYR tinha } \\
\text { concentrações de sangue do cordão abaixo do percentil } 2,5 \text { para cálcio } \\
(19 \% \text { versus } 2 \%) \text {, zinco }(13 \% \text { versus } 3 \%) \text {, ferro (19\% versus } 2 \%) \text { e } \\
\text { vitamina A (13\% versus } 3 \%) \text {. }\end{array}$ \\
\hline $\begin{array}{r}\text { ALI H, et } \\
(2017)\end{array}$ & $\begin{array}{l}\text { Examinar os efeitos independentes e } \\
\text { combinados da suplementação pré-natal e } \\
\text { neonatal com vitamina A na função } \\
\text { cognitiva de crianças aos } 8 \text { anos de idade. }\end{array}$ & 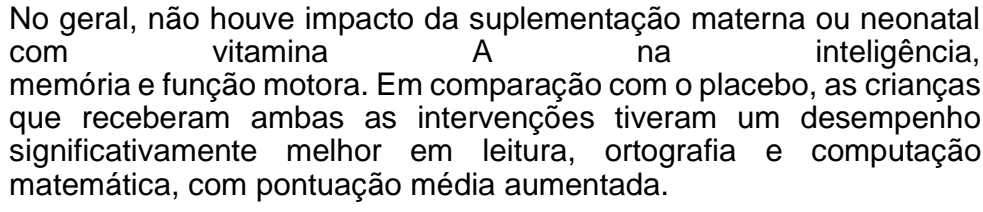 \\
\hline $\begin{array}{c}\text { CRUZ S, et al. } \\
\text { (2017). }\end{array}$ & $\begin{array}{l}\text { Comparar o estado nutricional da vitamina A } \\
\text { por trimestre de gestação, bem como avaliar } \\
\text { sua influência no IMC pré-gestacional, } \\
\text { ganho de peso gestacional total e presença } \\
\text { de anemia em mulheres submetidas à } \\
\text { Derivação Gástrica em Y de Roux (DGYR). }\end{array}$ & $\begin{array}{l}\text { A deficiência de vitamina A na gravidez afetou } 90 \% \text { das mulheres, } \\
86,7 \% \text { desenvolveram cegueira noturna e } 82,8 \% \text { apresentaram } \\
\text { deficiência de vitamina A leve. Das mulheres avaliadas, } 73,3 \% \\
\text { desenvolveram anemia na gravidez, sendo que } 90,9 \% \text { apresentavam } \\
\text { deficiência de vitamina A e } 86,4 \% \text { cegueira noturna. }\end{array}$ \\
\hline $\begin{array}{l}\text { CRUZ S, et al. } \\
\text { (2018). }\end{array}$ & $\begin{array}{l}\text { Comparar o estado nutricional de vitamina } A \\
\text { em mulheres que foram submetidas por } \\
\text { Derivação Gástrica em } Y \text { de Roux (DGYR), } \\
\text { que se tornaram gravidas (G1), ou não (G2) } \\
\text { considerando o mesmo intervalo de tempo } \\
\text { pós cirúrgico. Também, buscou-se } \\
\text { comparar os impactos da deficiência de } \\
\text { vitamina A em ambos, mãe e infantes. }\end{array}$ & $\begin{array}{l}\text { Em G1, a deficiência de } \beta \text {-caroteno se mostrou maior do que a } \\
\text { deficiência de retinol nos } 3 \text { períodos estudados: Antes da cirurgia (T0); } \\
\leq 1 \text { anos após a cirurgia (T1); } \leq 2 \text { anos após a cirurgia (T2). No período } \\
\text { T1, G1 apresentou sua maior deficiência de } \beta \text {-caroteno, equivalente a } \\
77 \% \text {. Os níveis de retinol se mantiveram com variações similares nos } \\
\text { três períodos. A presença de cegueira noturna no G1 foi observada } \\
\text { nos } 3 \text { períodos avaliados, com maior intensidade no T1. Em relação ao } \\
\text { nascimento, foi percebido prematuridade apenas naquelas mães } \\
\text { geraram o neonato antes de completar } 1 \text { ano de cirurgia. Porcentagem } \\
\text { de menor peso por idade (SGA) foi similar em ambos os períodos (T1 } \\
\text { e T2). }\end{array}$ \\
\hline $\begin{array}{l}\text { GIMENES JC, } \\
\text { et al. (2018). }\end{array}$ & $\begin{array}{l}\text { Avaliar o perfil nutricional de crianças de } \\
\text { mães que foram submetidas ao } \\
\text { procedimento cirúrgico bypass gástrico } \\
\text { previamente a gestação. }\end{array}$ & $\begin{array}{l}\text { Dos } 13 \text { neonatos envolvidos no estudo, } 30,7 \% \text { apresentaram } \\
\text { deficiência de vitamina A. Adicionalmente, foi identificado níveis } \\
\text { inadequados de outros micronutrientes como cálcio, zinco, ácido fólico } \\
\text { e vitamina B12. }\end{array}$ \\
\hline
\end{tabular}

REAS | Vol.13(12) | DOI: https://doi.org/10.25248/REAS.e9521.2021

\section{CONCLUSAO}

A gravidez após DGYR pode representar uma situação de alto risco para DVA, que está entre as deficiências de vitaminas mais prevalentes e tem grandes impactos na mãe e saúde infantil.

Gestantes pós DGYR tiveram um aumento na taxa de neonatos pequenos para a idade gestacional em comparação com neonatos de mães saudáveis, além de deficiências de vitaminas e oligoelementos.

A inteligência geral, memória e função motora não foram afetadas pelo pré-natal

suplementação do recém-nascido com vitamina $A$, mas o desempenho escolar melhorou quando as duas intervenções foram fornecidas. Os resultados mostram que a suplementação de vitamina $A$ também pode fornecer benefício sem efeitos adversos.

Importância do acompanhamento do estado nutricional de vitamina A no pré-natal desde o início da gestação para mulheres que se submeteram a DGYR, tendo em vista a alta porcentagem de deficiência de vitamina $A$ e cegueira noturna encontrados a partir do primeiro trimestre da gestação.

O déficit de vitamina $A$ no período pós-cirúrgico foi intensificado pela gravidez. Além disso, percentuais intensificado pela gravidez. Além disso, percentuais observados em ambos os tempos estudados.

Neonatos de mães que passaram pelo procedimento bypass gástrico previamente a gestação apresentaram deficiência importante de vitamina A (além de inadequação de outros nutrientes, principalmente 0 ferro). 


\begin{tabular}{|c|c|}
\hline AUTORIA & OBJETIVO \\
\hline $\begin{array}{c}\text { CRUZ S, et al. } \\
\text { (2018). }\end{array}$ & $\begin{array}{l}\text { Avaliar a inadequação do estado nutricional } \\
\text { de vitamina A causada pela gravidez, pela } \\
\text { Derivação Gástrica em Y de Roux (DGYR), } \\
\text { ou pela ocorrência gravidez e DGYR } \\
\text { concomitantemente, } \\
\text { bem como o impacto desta deficiência } \\
\text { vitamínica na mãe e saúde da criança. }\end{array}$ \\
\hline $\begin{array}{l}\text { DEMINICE } \\
\text { TMM, et al. } \\
(2018) .\end{array}$ & $\begin{array}{l}\text { Examinar a ingestão de vitamina A das } \\
\text { mães e as concentrações de retinol no } \\
\text { sangue materno, leite materno e cordão } \\
\text { umbilical de recém-nascidos; descrever a } \\
\text { ingestão de vitamina A no último mês de } \\
\text { gestação e as concentrações de retinol no } \\
\text { sangue materno e do recém-nascido e no } \\
\text { leite materno; e analisar as associações } \\
\text { entre essas variáveis. }\end{array}$ \\
\hline $\begin{array}{l}\text { JOHNSON LM, } \\
\text { et al. (2019). }\end{array}$ & $\begin{array}{l}\text { Investigar a prevalência de deficiências de } \\
\text { vitaminas } A, B 1, B 12, D \text { e folato em } \\
\text { pacientes com gastrectomia vertical (GV) e } \\
\text { derivação gástrica em Y de Roux (DGYR) } \\
\text { em uma única instituição. }\end{array}$ \\
\hline $\begin{array}{c}\text { CHEN B, et al. } \\
(2020) .\end{array}$ & $\begin{array}{l}\text { Investigar os efeitos da deficiência de } \\
\text { vitamina A durante diferentes períodos de } \\
\text { vida na estrutura da microbiota da mucosa } \\
\text { colônica em ratos adolescentes. }\end{array}$ \\
\hline $\begin{array}{c}\text { MESQUITA } \\
\text { LMS, et al. } \\
(2021) .\end{array}$ & $\begin{array}{l}\text { Resumir de forma abrangente os dados } \\
\text { atuais e mostrar o impacto potencial da } \\
\text { vitamina A e seus carotenoides provitamina } \\
\text { no estado nutricional durante a gravidez e a } \\
\text { lactação. }\end{array}$ \\
\hline $\begin{array}{l}\text { TAN M, et al. } \\
(2021) .\end{array}$ & $\begin{array}{l}\text { Avaliar se a deficiência de vitamina } A \\
\text { induzida pela dieta materna durante a } \\
\text { gravidez pode causar alterações no sistema } \\
\text { nervoso entérico que levam à disfunção } \\
\text { gastrointestinal na prole de ratos. }\end{array}$ \\
\hline
\end{tabular}

Fonte: Cotillo THC, et al., 2021.

\section{PRINCIPAIS RESULTADOS}

A DGYR, por si só, teve maior impacto na inadequação de retinol, $\beta$ caroteno e no aumento do percentual de cegueira noturna quando comparado a gestantes não cirúrgicas. Quando a cirurgia foi associada à gravidez, notou-se mais de $75 \%$ de inadequação de retinol e $\beta$ caroteno, além de maior percentual de indivíduos com cegueira noturna. O grupo de gestantes que realizaram DGYR também apresentou razão de prevalência aumentada para o desenvolvimento de intercorrências gestacionais e neonatais, quando comparado ao grupo de gestantes que não realizaram a cirurgia.

Concentrações de retinol $<0,70 \mu \mathrm{mol} / \mathrm{L}$ foram encontradas em $57,2 \%$ das amostras de sangue materno e em $94,9 \%$ das amostras de cordão umbilical. Um total de $27,9 \%$ das amostras de leite materno apresentara concentrações de retinol $<1,05 \mu \mathrm{mol} / \mathrm{L}$. A ingestão média de vitamina $A$ foi inadequada em $44,7 \%$ das mães.

A deficiência de vitamina $A$ foi incomum no pré-operatório $(2,7 \%$ gastrectomia (GV) versus 1,7\% DGYR), mas aumentou após a cirurgia (9,4\% GV versus 15,9\% DGYR dentro de 1 ano pós-operatório e 5,2\% GV versus $7,7 \%$ DGYR após 1 ano).

A deficiência de vitamina A (DVA) em diferentes períodos de vida pode alterar o microbioma intestinal em ratos, mas DVA nos primeiros períodos de vida (especialmente gestação e / ou lactação) leva a uma períodos de vida (especialmente gestação e / ou lactação) leva a uma bem como a um desequilíbrio da proporção entre Firmicutes e Bacteroidetes

Apesar do papel significativo dos carotenoides e vitamina A no estado nutricional de grávidas e mulheres lactantes, uma porcentagem considerável de mulheres globalmente não consome uma quantidade considerável de mulheres globa
suficiente destes nutrientes.

Os dados revelaram reduções marcantes nas densidades médias de neurônios entéricos colinérgicos e nitrérgicos no cólon e disfunção gastrointestinais evidenciada por flatulência intestinal leve, aumento do conteúdo de água fecal, tempo de trânsito gastrointestinal total prolongado e motilidade cólica reduzida em filhos adultos do grupo deficiência de vitamina $A$.

\section{CONCLUSAO}

A DGYR isoladamente proporciona maior impacto negativo no estado nutricional da vitamina $A$ em comparação com a gestação. Quando a gestação ocorre após a cirurgia, pode resultar em riscos ainda maiores para a inadequação sérica de retinol e $\beta$-caroteno, além da ocorrência de cegueira noturna, o que intensifica as intercorrências gestacionais e neonatais.

Observada alta porcentagem de mulheres grávidas com ingestão inadequada de vitamina A durante o mês anterior ao parto. Mães com concentrações médias insuficientes de retinol sérico deram à luz a neonatos com concentrações mais baixas deste micronutriente no cordão umbilical.

A prevalência de deficiências de vitaminas, tanto no pré quanto no pós-operatório, foi menor em nossa população de pacientes de cirurgia bariátrica do que em outros relatos.

A deficiência de vitamina $A$ no início da vida pode aumentar patógenos oportunistas que causam infecção. Portanto, o estágio inicial da vida pode se tornar uma janela de oportunidade para intervenção da vitamina $A$, melhorando a microbiota intestinal causada pela deficiência de vitamina $A$, mas o mecanismo específico requer pesquisas mais aprofundadas.

$O$ estado nutricional materno é um dos mais fatores importantes que desencadeiam a programação fetal, e vitamina $A$ e seus carotenoides provitamina $A$ são moléculas importantes que auxiliam na concepção, implantação, placentação e crescimento fetal regular.

A suplementação de vitamina A durante o início da gravidez pode prevenir problemas gastrointestinais induzidos por deficiência de vitamina A. Portanto, deficiência de vitamina $A$ pré-natal pode ser um dos fatores de risco ambientais para disfunção gastrointestinal em descendentes de ratos. 


\section{DISCUSSÃO}

\section{Deficiência de vitamina A após DGYR}

A DGYR é comumente adotada como procedimento cirúrgico para redução da gordura corporal em casos de obesidade, sendo considerada técnica "padrão ouro". Contudo, os indivíduos que se submetem a essa técnica acabam desenvolvendo deficiências nutricionais, devido a redução da anatomia intestinal responsável pelo trânsito e absorção de alimentos (JOHNSON LM, et al., 2019).

Deficiências de vitaminas lipossolúveis, tal como a vitamina $A$ é bastante recorrente após a cirurgia bariátrica. Isso se deve a reconfiguração anatômica do trato gastrointestinal que culmina em uma menor digestão de lipídeos dietéticos (importante para a absorção de vitamina A) (QUEIROZ NA, et al., 2020).

Pesquisa realizada entre abril de 2012 e abril de 2016 analisou o nível plasmático de vitamina A pré e pósoperatório de pacientes submetidos a DGYR. Esses pacientes eram acompanhados por nutricionistas e consumiam suplemento que garantisse pelo menos $200 \%$ da necessidade diária de vitamina A tanto no pré quanto no pós-cirúrgico. Participaram 110 pacientes e os dados pré-operatórios foram obtidos de 60 pacientes, pós-operatórios ( $\leq 1$ ano) de 69 e pós-operatório ( $>1$ ano) de 52 . Foi demonstrado que a deficiência de vitamina $A$ foi incomum no pré-operatório (1,7\%), porém aumentou para $15,9 \%$ dentro de um ano pós-operatório e após um ano diminuiu para 7,7\% (JOHNSON LM, et al., 2019).

Estudo realizado no Rio de Janeiro avaliou 30 gestantes pós DGYR que seguiram um protocolo de suplementação diária de vitaminas e minerais contendo $5.000 \mathrm{UI}$ de acetato de retinol e registrou que a deficiência de vitamina A se apresentou em $90 \%$ das gestantes, sendo leve em $82,8 \%$ do total de casos e menos presente no primeiro trimestre de gestação. Foi encontrada uma associação entre a inadequação sérica do retinol e a presença de cegueira noturna no primeiro e segundo trimestres nas mulheres com deficiência de vitamina A leve. Dentre as mulheres que desenvolveram deficiência de vitamina A gestacional, $88,9 \%$ demonstraram cegueira noturna. Além disso, $100 \%$ das mulheres que estavam acima da faixa de peso recomendada apresentaram o quadro de cegueira noturna, enquanto naquelas com ganho de peso adequado ou abaixo da faixa recomendada o percentual de cegueira noturna encontrado foi de $80 \%$ e $86,7 \%$, respectivamente (CRUZ S, et al., 2017).

O mesmo foi concluído em outro estudo realizado no Rio de Janeiro. As participantes foram divididas em dois grupos: grupo $1 \mathrm{com} 80$ gestantes e grupo 2 com 40 gestantes pós DGYR. Um dos critérios de exclusão para o grupo 2 era uso de suplementos contendo vitamina $A$ durante a gestação. Os pesquisadores observaram que gestantes do grupo 2 apresentaram 9,1 vezes mais chances de desenvolver deficiência de vitamina $A$, considerando os níveis de retinol sérico, e 23,7 vezes, considerando níveis séricos de $\beta$-caroteno, quando comparadas a gestantes do grupo 1, concluindo que a gestação após a DGYR pode representar uma situação de alto risco para deficiência. Também se observou uma porcentagem de $75 \%$ de cegueira noturna para gestantes do grupo 2 e de 20\% em gestantes do grupo 1 (MACHADO SN, et al., 2016).

A DGYR por si só proporciona maior impacto negativo no estado nutricional da vitamina $A$ do que a gestação, quando combinadas, os riscos são ainda maiores. Isso foi o que concluiu um estudo realizado no Brasil que dividiu as participantes em três grupos: grupo $1 \mathrm{com} 80$ gestantes que não realizaram DGYR, grupo 2 com 40 gestantes pós DGYR e grupo 3 com 77 mulheres não gestantes que realizaram DGYR. As mulheres que realizaram DGYR estavam com suplementação de rotina de $5000 \mathrm{UI}$ de acetato de retinol. Foi observado que as mulheres do grupo 2 apresentaram porcentagem de inadequação de retinol três vezes maior quando comparado as mulheres dos grupos 1 e 3 . Além disso, as mulheres do grupo 2 apresentaram médias de retinol e $\beta$-caroteno significativamente abaixo das encontradas no grupo 3. O mesmo se observa em relação a cegueira noturna, foi observado que a porcentagem de mulheres do grupo $2 \mathrm{com}$ cegueira noturna foi maior do que as dos grupos 1 e 3, 75\%, 20\% e 40\% respectivamente (CRUZ S, et al., 2018).

Estudo com 30 gestantes que realizaram DGYR e que estavam suplementando $5000 \mathrm{UI}$ de retinol por dia, observaram que a deficiência de vitamina $A$, considerando nível de retinol sérico inadequado, se apresentou no primeiro trimestre de gestação em $63 \%$ das gestantes. No segundo trimestre a porcentagem se manteve, já no terceiro trimestre aumentou para $66 \%$. Casos de deficiência de vitamina A moderados foram observados 
apenas no primeiro trimestre de gestação, no segundo e terceiro trimestre foram observados casos leves. Além dos níveis inadequados de retinol, as gestantes apresentaram níveis séricos inadequados de $\beta$ caroteno, sendo $80 \%$ no primeiro trimestre, $67 \%$ no segundo e $73 \%$ no terceiro. Portanto a inadequação do $\beta$-caroteno foi maior do que a observada para o retinol. A cegueira noturna foi detectada em $57 \%$ das mulheres em cada trimestre durante a pesquisa. Percebeu-se, também, uma associação ente deficiência de vitamina $A$ à infecção do trato urinário e síndrome de dumping (CHAGAS C, et al., 2016).

Ao se comparar os valores de vitamina $A$ antes da cirurgia (T0), até se completar um ano (T1) e 2 anos de cirurgia (T2), em 77 mães que foram previamente submetidas a DGYR, percebe-se que a deficiência de $\beta$ caroteno é mais expressiva que a de retinol em todos os três períodos estudados. Além disso, a deficiência $\beta$-caroteno se mostrou significantemente maior no período T1 em comparação ao período T2 (77,1 vs. 45,8\%, respectivamente). Também foi percebido um maior percentual de cegueira noturna em T1 (54,2\%) em relação a T2 (41,7\%). O estudo também mostrou dados correspondente a variáveis de nascimento, sendo identificado um mais elevado percentual de UTI no período T1 $(57,1)$ em relação a T2 $(28,1 \%)$ e prematuridade apenas naquelas mães geraram o neonato em T1 (CRUZ S, et al., 2018).

A DGYR materna está associada a deficiências nutricionais no neonato. Um estudo francês avaliou 56 gestantes que não realizaram cirurgia (grupo controle) e 56 gestantes pós DGYR que suplementavam 1200 $\mu \mathrm{g}$ de vitamina A diariamente e concluiu que os níveis de vitamina A no sangue do cordão umbilical de recémnascidos de mães pós DGYR foi significativamente menor quando comparados ao grupo controle (GASCOIN G, et al., 2017).

Em território brasileiro o cenário é similar, um estudo recente realizado em Ribeirão Preto coletou amostras de sangue de 180 gestantes, leite materno de 154 gestantes e cordão umbilical de 159 recém-nascidos e registrou baixos níveis de retinol em $57,2 \%$ amostras de sangue, $27,9 \%$ de leite materno e $95 \%$ de cordão umbilical. Foi avaliado também a ingestão de vitamina $A$ das gestantes através de um questionário quantitativo. $O$ consumo médio foi calculado individualmente e caso a gestante relatasse ingestão de suplemento de vitamina $\mathrm{A}$, a quantidade fornecida pelo suplemento era somada à obtida com os alimentos consumidos. Foram obtidos 179 questionários e observado níveis inadequados de retinol em 44,7\% dos questionários. Essa baixa ingestão pode explicar o fato de mais da metade das gestantes terem baixos níveis séricos de vitamina A (DEMINICE TMM, et al., 2018).

\section{Efeitos da vitamina A no feto}

Durante o período de gestação, é de suma importância a suprir demandas nutricionais tanto em prol da saúde materna quanto do feto. A vitamina A é um micronutriente essencial para o desenvolvimento fetal uma vez que está relacionada com divisão e diferenciação celular do feto, manutenção do epitélio tanto da mãe quando do bebê, desenvolvimento e maturação de órgãos e ossos do embrião, contribuição para o sistema imune de ambos contra patógenos e funcionalidade ocular para visão noturna da mãe (MAIA SD, 2019; YONEKURA L, et al., 2020).

Entretanto, a hipovitaminose A durante o período gestacional é bastante comum, e tal carência nutricional deve ser controlada, pois o feto depende exclusivamente do retinol circulante da mãe para seu desenvolvimento. No terceiro trimestre de gestação a demanda da vitamina $\mathrm{A}$ aumenta, pois há um aumento fisiológico dos níveis de sangue na gestante e aumento da velocidade do desenvolvimento fetal (DEMINICE TMM, et al., 2018). Em estágios mais avançados do desenvolvimento embrionário pode-se perceber a relação da deficiência de vitamina A malformação do coração, sistema nervoso e suas estruturas derivadas, sistema urogenital, sistema circulatório, e sistema respiratório, esqueletos e vísceras (ZILE MH, 2001).

Em estudo conduzido em 1180 mulheres grávidas em seus últimos trimestres de gestação mostrou que 48 recém-nascidos que apresentaram malformação congênita e suas respectivas mães havia deficiências em alguns micronutrientes, como vitaminas A, E, B12, B9, selênio, zinco e magnésio (HAMMOUDA SA, et al., 2013).

Em pesquisas realizadas em modelo animal, percebe-se algumas alterações na formação do embrião por conta da hipovitaminose já no início da gestação. A vitamina A crucial para a formação do coração primitivo 
e sistema circulatório, sendo assim, o déficit da mesma pode resultar em severas anormalidades incluindo a morte do embrião (MAIA SD, 2019). Ademais, pode-se observar que déficit de vitamina $A$ em ratas grávidas em até duas semanas antes e durante a gestação pode também proporcionar malefícios a formação da rede nervosa fetal (HUANG Y e ZHENG, 2011). Outros prejuízos apontados em estudos em animais mostraram inadequada formação da parte interna do ouvido (FRENZ DA, et al., 2010) e desenvolvimento irregular do pâncreas endócrino (MATTHEWS KA, et al., 2004).

A malformação renal oriunda da hipovitaminose A também foi observada em pesquisas em humanos. Em um estudo em que se comparou gestantes deficientes em vitamina A e gestantes com níveis suficientes da vitamina, foi possível perceber que os recém-nascidos de mães deficientes apresentaram um rim significativamente menor em tamanho e baixos níveis de retinol no cordão umbilical, quando comparados aos recém-nascidos de mães com níveis suficientes da vitamina (EL-KHASHAB, 2013).

Embora os estudos em sobre deficiência de vitamina A em gestantes pós DGYR ainda sejam escassos, pode-se entender que a deficiência de vitamina $A$ no feto pode ser intensificada caso a mãe tenha passado pelo procedimento cirúrgico previamente. Gimenes JC, et al. (2018) reportou-se uma deficiência de vitamina A em $37 \%$ dos neonatos avaliados no estudo $(n=13)$, sendo eles de mães que foram submetidas previamente a bypass gástrico.

A nutrição materna é um fator importante na programação fetal. A vitamina $A$ e os carotenoides provitamina A (principalmente o $\beta$-caroteno) auxiliam na concepção, implantação, placentação e crescimento fetal regular. Desempenham um papel importante na prevenção de várias complicações gestacionais e em alterações epigenéticas (MESQUITA LMS, et al., 2021).

Em relação a microbiota intestinal, estudos em ratos sugeriram que a deficiência de vitamina A pode alterar a sua homeostase. Concluindo que a hipovitaminose $A$ no início da vida (gestação e lactação) pode aumentar patógenos oportunistas que podem causar infecções (CHEN B, et al., 2020).

Muitos distúrbios gastrointestinais são causados pela formação anormal do sistema nervoso entérico e a deficiência de vitamina A durante a gestação afeta vários processos de desenvolvimento do sistema nervoso central durante a embriogênese e a vida fetal. Ratas gestantes foram induzidas a deficiência de vitamina $A$ através de dieta e houve um aumento no risco de disfunção gastrointestinal na prole. Foram observadas reduções marcantes nas densidades médias de neurônios entéricos colinérgicos e nitrérgicos no cólon e disfunção gastrointestinal evidenciada por flatulência intestinal leve, aumento do conteúdo de água fecal, tempo de trânsito gastrointestinal prolongado e motilidade do cólon reduzida em filhos adultos do grupo com deficiência de vitamina A. A suplementação de vitamina A no início da gestação pode evitar esses problemas gastrointestinais (TAN M, et al., 2021).

O desempenho escolar de crianças melhorou quando a vitamina $A$ foi suplementada no período pré-natal e neonatal, porém a inteligência geral, memória e as funções motoras não foram afetadas pela suplementação. Estudo realizado em Bangladesh, país situado no centro-sul da Ásia, avaliou crianças de 8 anos que haviam participado de 2 estudos randomizados controlados com placebo anteriormente. No primeiro ensaio, as mães das crianças foram suplementadas com vitamina $A$ ou $\beta$-caroteno ou placebo desde o início da gestação até 3 meses após o parto; e no segundo ensaio, as crianças nascidas dessas mães receberam um placebo ou um suplemento de vitamina A de 50.000 UI no nascimento (ALI H, et al., 2017).

\section{CONSIDERAÇÕES FINAIS}

A DGYR é um procedimento que aumenta a ocorrência de deficiência de vitamina $A$, devido as alterações anatômicas e fisiológicas do procedimento cirúrgico, contudo, quando associado com a gestação, há um agravamento do quadro podendo ocorrer efeitos adversos na mãe e no feto. Notificou-se que uma prevalência alta cegueira noturna em gestantes pós DGYR. Em relação ao desenvolvimento fetal, se reportou que a deficiência da vitamina pode corroborar para a má formação congênita de múltiplos órgãos. O estudo colabora para maiores conhecimentos sobre a importância do acompanhamento nutricional pré-cirúrgico, pós-cirúrgico e durante a gestação é necessário para monitorar essa deficiência, sendo possível sua correção o mais breve possível. 


\section{REFERÊNCIAS}

1. AGARWAI R, et al. Vitamin A status of low and normal birth weight infants at birth and in early infancy. Indian Journal of Pediatrcs, 2013; 50(10): 951-953.

2. AKHTER Z, et al. Pregnancy after bariatric sugery and adverse perinatal outcomes: A systematic review and meta-analysis. PLoS Medicine, 2019; 16(8): e1002866.

3. ALI H, et al. Effect of maternal antenatal and newborn supplementation with vitamin $A$ on cognitive development of schoolaged children in rural Bangladesh: a follow-up of a placebo-controlled, randomized trial. The American Journal of Clinical Nutrition, 2017; 106(1): 77-87.

4. BELLVER J, et al. Female obesity impairs in vitro fertilization outcome without affecting embryo quality. Fertility and Sterility, 2010; 93(2): 447-54.

5. BROUGHTON DE, MOLEY KH. Obesity and female infertility: potential mediators of obesity's impact. Fertility and Sterility, $2017 ; 107(4)$.

6. CHAGAS C, et al. Vitamin A status and its relationship with serum zinc concentrations among pregnant women who have previously undergone Roux-en-Y gastric bypass. International Journal of Gynecology \& Obstetrics, 2016; 133(1): 94-7.

7. CHECKLEY W, et al. Maternal Vitamin A supplementation and lung function in offspring. New England Journal of Medicine, 2010; 362(19): 784-1794.

8. CHEN B, et al. Vitamin A deficiency in the early-life periods alters a diversity of the colonic mucosal microbiota in rats. Frontiers in Nutrition, 2020; 7: 580780.

9. CRUZ S, et al. Comparative study of the nutritional status of vitamin A in pregnant women and in women who became pregnant or did not after Roux-en-Y gastric bypass. Nutrición Hospitalaria, 2018; 35(2): 421-427.

10. CRUZ S, et al. Relationship between the nutritional status of vitamin A per trimester of pregnancy with maternal anthropometry and anemia after Roux-en-Y Gastric Bypass. Nutrients, 2017; 9(9): 989.

11. CRUZ S, et al. Roux-en-Y Gastric Bypass Aggravates Vitamin A Deficiency in the Mother-Child Group. Obesity Surgery, 2018; 28(1): 114-121

12. DEMINICE TMM, et al. Vitamin A intake of Brazilian mothers and retinol concentrations in maternal blood, human milk, and the umbilical cord. Journal of International Medical Research, 2018; 46(4): 1555-1569.

13. CUESTA M, et al. Fat-soluble vitamin deficiencies after bariatric surgery could be misleading if they are not appropriately adjusted. Nutrición Hospitalaria, 2014; 30(1): 118-23.

14. EL-KHASHAB EK, et al. Effect of maternal vitamin A deficiency during pregnancy on neonatal kidney size. Journal of Perinatal Medicine, 2013; 41(2): 199-203.

15. FRENZ, DA, et al. Retinoid signaling in inner ear development: A "Goldilocks" phenomenon. American Journal of Medical Genetics, 2010; 152A: 2947-2961.

16. GASCOIN G, et al. Risk of low birth weight and micronutrient deficiencies in neonates from mothers after gastric bypass: a case control study. Surgery for Obesity and Related Diseases, 2017; 13(8): 1384-1391.

17. GIMENES JC, et al. Nutritional Status of Children from Women with Previously Bariatric Surgery. Obesity Surgery, 2018; 28(4): 990-995.

18. HAMMOUDA SA, et al. Serum levels of some micronutrients and congenital malformations: a prospective cohort study in healthy saudi-arabian first-trimester pregnant women. International Journal for Vitamin and Nutrition Research, 2013; 83(6): 346-354.

19. HARRISON RK, et al. Managing malnourishment in pregnancy after bariatric surgery. Journal of Obstetrics and Gynaecology Canada, 2018; 40(2): 211-214.

20. HUANG Y, ZHENG S. The effect of vitamin A deficiency during pregnancy on anorectal malformations. Journal of Pediatric Surgery, 2011; 46(7): 1400-1405.

21. JOHNSON LM. Analysis of vitamin levels and deficiencies in bariatric surgery patients: a single-institutional analysis. Surgery for Obesity and Related Diseases, 2019; 15(7): 1146-1152.

22. LEE R, et al. A Review of the impact of bariatric surgery in Women with polycystic ovary syndrome. Cureus, 220; 12(10): e10811.

23. MACHADO SN, et al. Influence of Roux-en-Y Gastric Bypass on the nutritional status of vitamin A in pregnant women: a comparative study. Obesity Surgery, 2016; 26(1): 26-31.

24. MAIA SB, et al. Vitamin A and pregnancy: A Narrative Review. Nutrients, 2019; 11(3): 681.

25. MARTINS APR, MASQUIO DCL. O papel da vitamina A na saúde materno-fetal: uma revisão bibliográfica. Revista Eletrônica Acervo Saúde, 2019; (20): e518.

26. MATTHEWS KA, et al. Vitamin A deficiency impairs fetal islet development and causes subsequent glucose intolerance in adult rats. Journal of Nutrition, 2004; 134(8): 1958-1963.

27. MESQUITA LMS, et al. The role of vitamin $A$ and its pro-vitamin carotenoids in fetal and neonatal programming: gaps in knowledge and metabolic pathways. Nutrition Reviews, 2021; 79(1): 76-87.

28. MITCHELL BG, GUPTA N. Roux-en-Y Gastric Bypass. StatPearls, 2020.

29. NCD Risk Factor Collaboration (NCD-RisC). Trends in adult body-mass index in 200 countries from 1975 to 2014: a pooled analysis of 1698 population-based measurement studies with 19.2 million participants. Lancet, 2016; 387:1377-1396.

30. QUEIROZ NA, et al. Cirurgia bariátrica e aspectos gerais sobre microbiota intestinal. In: COZZOLINO S. Biodisponibilidade de Nutrientes. 6- ed. Barueri: Manole, 2020;655-682p.

31. SILVESTRIS E, et al. Obesity as disruptor of the female fertility. Reproductive Biology and Endocrinology, 2018; $16(1): 22$.

32. SOCIEDADE BRASILEIRA DE CIRURGIA BARIÁTRICA E METABÓLICA (SBCBM). 2017. A cirurgia bariátrica. Disponível em: https://www.sbcbm.org.br/a-cirurgia-bariatrica/. Acessado em: 13 mai. de 2020.

33. TAN M, et al. Maternal vitamin A deficiency impairs cholinergic and nitrergic neurons, leading to gastrointestinal dysfunction in rat offspring via RARß. Life Sciences, 2021; 264: 118688.

34. TIMONEDA J, et al. Vitamin A Deficiency and the Lung. Nutrients, 2018; 10 (9)

35. WELBOURN R, et al. Bariatric Surgery Worldwide: Baseline Demographic Description and One-Year Outcomes from the Fourth IFSO Global Registry Report 2018. Obesity Surgery, 2019; 29(3): 782-795.

36. WISE LA, et al. Body size and time-to-pregnancy in black women. Human Reproduction, 2013; 28(10): $2856-2864$.

37. WORLD HEALTH ORGANIZATION (WHO). 2020. Obesity and Overweight. Disponível em: https://www.who.int/en/newsroom/fact-sheets/detail/obesity-and-overweight. Acessado em: 05 de mai. de 2020.

38. YONEKURA L, et al. Vitamina A (retinol) e carotenoides. In: COZZOLINO, S. Biopdisponibilidade de Nutrientes. 6 ed. Barueri (SP): Manole, 2020. p. $173-195$

39. ZILE MH. Function of vitamin A in vertebrate embryonic development. J. Nutr. 2001, 131, 705-708. 